\title{
Les permissionnaires du front face aux cheminots pendant la Première Guerre mondiale
}

World War I soldiers on leave's attitude toward railwaymen

\section{Emmanuelle Cronier}

\section{OpenEdition}

\section{Journals}

Édition électronique

URL : https://journals.openedition.org/rhcf/101

DOI : $10.4000 /$ rhcf.101

\section{Éditeur}

Rails \& histoire

Édition imprimée

Date de publication : 1 décembre 2007

Pagination : 91-105

ISSN : 0996-9403

Référence électronique

Emmanuelle Cronier, « Les permissionnaires du front face aux cheminots pendant la Première Guerre mondiale », Revue d'histoire des chemins de fer [En ligne], 36-37 | 2007, mis en ligne le 10 mai 2011, consulté le 22 avril 2022. URL : http://journals.openedition.org/rhcf/101 ; DOI : https://doi.org/ 10.4000/rhcf.101 


\title{
Les permissionnaires du front face aux cheminots pendant la Première Guerre mondiale
}

\author{
Emmanuelle Cronier \\ Docteur de l'université de Paris I - Panthéon-Sorbonne
}

Les permissionnaires du front, ces combattants qui obtiennent à partir de juillet 1915 quelques jours de congé à passer dans leur famille avant de retourner au front, sont par essence des voyageurs, entre le front et l'arrière ; la mort et la vie. Tout le sens de la permission tient dans ce passage d'un monde à l'autre, dont le milieu ferroviaire constitue à la fois un instrument et une étape. Le transport des permissionnaires du front occidental s'inscrit dans la vaste mobilisation des chemins de fer pendant la Première Guerre mondiale, puisque c'est essentiellement par train qu'ils rentrent chez eux et retournent au front ensuite ${ }^{1}$. Le regard que la communauté combattante porte sur les cheminots, artisans de leur voyage, nous est connu grâce à plusieurs types de sources, comme les «journaux des tranchées», les témoignages comme les mémoires ou les carnets de combattants, mais aussi par la littérature ou la chanson². Les sources militaires ou ferroviaires permettent parfois de saisir la confrontation des deux communautés. L'orientation de ma recherche, qui porte sur les permissionnaires, explique que le point de vue des cheminots n'apparaisse qu'en creux ici, alors qu'il serait possible de le saisir dans des sources comme La Tribune des cheminots, par exemple.

La confrontation des permissionnaires et des cheminots présente plusieurs intérêts. La rencontre est placée sous le signe de l'éphémère, tandis que le contexte psychologique du retour à l'arrière est totalement différent de celui de la mobilisation ou des nombreux transports de

1- À proximité du front, ils rejoignent les gares d'embarquement à pied, en camion dans certains cas, voire en automobile dans le cas des officiers.

2- Les «journaux de tranchées», appelés aussi «presse du front» depuis l'étude de Stéphane Audoin-Rouzeau (À travers leurs journaux: 14-18. Les combattants des tranchées, Armand Colin, 1986, 222 p.), font référence aux journaux rédigés par des mobilisés, mais le statut militaire de leurs rédacteurs et leurs conditions de rédaction et de diffusion sont très divers. En particulier, beaucoup ne sont ni rédigés au front, ni par des combattants. C'est pourquoi le titre de «journaux de tranchées» doit, à mon avis, toujours être utilisé avec des guillemets soulignant cette diversité. 
troupes qui amènent les combattants à croiser fréquemment le personnel ferroviaire. Dans quelle mesure cela se traduit-il dans les représentations et peut-on observer des différences selon que le combattant part ou revient de permission ? Par ailleurs, les cheminots appartiennent dans l'esprit des combattants à la catégorie des « embusqués » en tant qu'affectés spéciaux. Dans quelle mesure le regard et le comportement des permissionnaires traduisent-ils cette classification morale de la société en guerre? Enfin, le contexte ferroviaire est celui d'un transport de masse qui reste jusqu'à la fin de la guerre placé sous le signe de l'improvisation permanente. Les trains spéciaux de permissionnaires subissent plus que les autres convois militaires les effets de la crise des chemins de fer, dont ils cumulent tous les dysfonctionnements : lenteur, retard, matériel dégradé, mal éclairé et mal chauffé3. Dans quelle mesure cette situation influence-t-elle le discours et le comportement des permissionnaires à l'égard du personnel ferroviaire?

D’une manière plus générale, quelle identité des cheminots les combattants construisent-ils à la faveur de leur rencontre, lorsqu'ils sont permissionnaires? Comment les représentations issues du monde combattant articulent-elles l'utilité des cheminots pour les permissionnaires, liée à leurs compétences techniques, leurs responsabilités dans la crise ferroviaire et l'éloignement du risque dont ils bénéficient ?

Pendant la guerre, tous les cheminots ayant plus de six mois d'ancienneté sont mobilisés à leur poste dans le cadre d'une affectation spéciale, soit comme sapeurs du $5^{\mathrm{e}}$ Génie pour le personnel de la Voie et des réseaux, soit dans les sections des chemins de fer de campagne pour l'Exploitation ou l'entretien des voies. On trouve dans ces sections un peu moins de 20000 hommes, en net sous-effectif par rapport à l'avant-guerre dans un contexte d'augmentation massive du trafic ${ }^{4}$. Les permissionnaires sont amenés à croiser plusieurs catégories de personnel au cours de leur voyage entre le front et l'arrière : les mécaniciens, chauffeurs et conducteurs; le personnel du service Voie et Bâtiments (agents d'entretien et de signalisation, garde-barrières); enfin celui de

3- La crise des chemins de fer pendant la guerre est étudiée par Marcel Peschaud dans Les Chemins de fer pendant et depuis la guerre, Paris, Dunod, s.d., 202 p., p. 22-53. Voir aussi A. Marchand [inspecteur général de la Cie des Chemins de fer de l'Est], Les Chemins de fer de l'Est et la guerre de 1914-1918, Nancy, Berger-Levrault, 1924, 612 p. Parmi les études contemporaines Georges Ribeill, Les Cheminots en guerre 1914-1920. Les métamorphoses d'une corporation, Certes-ENPC, 1988, 480 p. ; La Vie du rail, hors série, 11 Novembre 1918-1968, 96 p.

4- Peschaud, op. cit., p. 54-56. 
l'Exploitation, au guichet ou à la manœuvre en gare. En particulier, c'est le personnel des compagnies qui assure, pour des raisons comptables, le contrôle des titres de permission en gare pour en détacher les coupons de transport gratuit qui seront ensuite remboursés par l'État. Ces agents sont donc responsables du tampon apposé sur les titres qui date le début et la fin des permissions et permet de repérer les permissionnaires qui ont grappillé des jours. Une fois repéré, le fraudeur est ensuite passible de sanctions disciplinaires qui vont de la suspension de la permission suivante au conseil de guerre en cas de désertion. En outre, la militarisation des chemins de fer se traduit par la présence dans les gares et les trains d'un personnel militaire affecté au service d'ordre : des territoriaux fournissent la garde de police des trains, tandis que, dans les gares, des plantons surveillent les issues et le mouvement des permissionnaires. Enfin, le commissaire militaire, un officier en charge de la régulation, chapeaute désormais le chef de gare.

Les permissionnaires peuvent connaitre l'affectation des cheminots par le biais des insignes placés sur les manches et le col des sous-officiers et hommes de troupe - une locomotive pour le $5^{\mathrm{e}}$ Génie, et deux rameaux entrecroisés autour d'une roue pour les autres -, et des brassards qui distinguent les différentes affectations. Pendant la guerre, chacun est exercé à cette lecture des signes de la mobilisation. Les cheminots ne sont pas pour autant individualisés par les combattants au moyen d'une dénomination propre, puisque l'argot des combattants n'invente pas de terme nouveau pour les désigner, mais reprend les termes antérieurs à la guerre qui renvoient en particulier au métier des mécaniciens ${ }^{5}$.

Le couple permissionnaire/cheminot constitue-t-il un élément important de la représentation de la permission, à la mesure du rôle joué par le transport ferroviaire dans le voyage des combattants?

Ce n'est pas le cas dans les représentations qui émanent de l'arrière, où les cheminots sont par exemple totalement absents des scénarios du vaudeville, des films ou des revues où la figure du permissionnaire est au contraire très présente. Cette absence s'explique d'une part par le poids de la censure, qui tolère la représentation des « embusqués » civils mais pas celle des mobilisés. D’autre part, les cheminots sont épargnés au même titre que tous ceux qui participent, d'une façon ou d'une autre, à l'effort de guerre, pour stigmatiser essentiellement les fils à papa embusqués dans les administrations parisiennes. Il faut noter en outre que la rencontre des permissionnaires et des cheminots présente peu de

5- Albert Dauzat, L'Argot de la guerre, Paris, Armand Colin, 1919, 295 p. 
perspectives pour l'intrigue : elle ne se prête pas aux scènes romantiques, tandis que les perspectives moralisatrices sont limitées. Les métiers sont d'ailleurs peu représentés sur scène d’une manière générale. Des articles sur le transport des permissionnaires sont publiés dans la presse illustrée comme L'Illustration, mais le personnel des compagnies en est absent. Un grand article sur les gares de rassemblement publié en août 1916 ignore les cheminots au profit des soldats et des femmes de la CroixRouge. Seul un officier du service des étapes, chargé de diriger les permissionnaires sur leur destination, est mentionné à l'occasion d'une description idyllique de la soumission des combattants, aux «flots tumultueux mais toujours disciplinés $»^{6}$. Les permissionnaires font l'objet de nombreuses chansons qui évoquent parfois le retard des trains, mais mettent rarement le personnel ferroviaire en cause, puisque seul le chef de gare est évoqué à deux reprises dans le corpus de 115 chansons sur lequel j'ai travaillé. Dans Ca! c'est la perme !, écrite par deux mobilisés, et dans Il ne l'est pas le chef de gare, visée par la censure en septembre 1916, qui évoque le boniment des permissionnaires à la garde-barrière ${ }^{7}$. Le chef de gare tient cependant une grande place dans la culture populaire musicale depuis qu'il a eu le malheur d'agiter un drapeau jaune pour signaler le départ des trains, un rôle qui explique la grande fortune de la chanson Il est content le chef de gare, chantée en 1912 par Mansuelle sur l'air de Il était un petit navire, mais qui doit sa célébrité à l'usage intensif qu'en firent les combattants de la Première Guerre mondiale sous le titre Il est cocu le chef de gare, au point que le journal britannique The Guardian signalait d'ailleurs encore en 2001 la chanson comme caractéristique de la culture française $^{8}$.

En revanche, les cheminots sont très présents dans les représentations issues du monde combattant, mais de manière inégale selon leur fonction. Le trajet des permissionnaires est fréquemment occulté dans les romans de guerre, qui confrontent l'expérience du combat et le séjour à l'arrière, si bien que les cheminots en sont largement absents. Par exception, Le Sang noir, de Louis Guilloux, publié en 1935, met en

6- «Dans une gare de rassemblement », L'Illustration, 12 août 1916.

7- Archives de la préfecture de Police de Paris (APP), BA 701, Ça! c'est la perme, mars 1918, paroles de P. Gonnet, musique de E. Brunswick, visée le 6 mars 1918 ; BA 712 Il ne l'est pas le chef de gare, paroles de Villars, visée le 16 septembre 1919.

8- Chansons ferroviaires. 1907-1947, La Vie du rail et SPEF, 1998 ; Mr Smallweed, «A time to faff », The Guardian, 15 décembre 2001. Dans La Bête humaine, le chef de gare Roubaud est cocu, tandis que la chanson de Jean Ferrat, Le Chef de gare est amoureux, ou celle de Georges Brassens, Le Cocu, contiennent des références à cette chanson. 
scène une révolte de permissionnaires dans la gare de Saint-Brieuc en 1917, qui correspond à des faits réels, grossis pour l'occasion'. Les journaux combattants et les mémoires de guerre que j'ai consultés sont font assez peu de place à la figure des cheminots. À l'inverse, certaines catégories du personnel ferroviaire sont très présentes dans la " presse du front », et fréquemment associées aux « embusqués », tels les gardes-voies civils (GVC), les employés de planton dans les gares, les chefs de gare et commissaires militaires. Autrement dit, le personnel technique est peu représenté au profit de celui chargé du contrôle et de la régulation.

L'attitude des permissionnaires à l'égard du personnel ferroviaire est marquée par un regard critique qui varie de l'humour troupier, déjà en place avant guerre dans les pratiques conscriptives, au renversement symbolique et ritualisé de l'ordre et de la discipline pesant sur les permissionnaires, lors de leur voyage entre le front et à l'arrière, qui se transforme parfois en violence pendant des périodes spécifiques ${ }^{10}$.

Dès l'hiver 1915, des rapports signalent que « des permissionnaires du front, ont, pendant leur voyage en chemin de fer, invectivé grossièrement des GVC chargés de la surveillance de la voie, et même lancé divers objets, pouvant causer des accidents $»^{11}$. La lenteur des convois favorise en effet l'interpellation du personnel posté le long des voies ou dans les gares. Le haut commandement préconise alors que des « recommandations formelles» soient faites à ce sujet à ceux qui partent en permission, car ces « graves fautes » sont passibles du Conseil de guerre. Cependant, au cours de l'année 1916, le chahut tend à se ritualiser, en particulier dans les gares. Le jeune Louis Désalbres le raconte dans son carnet son voyage de Saint-Just-en-Chaussée à Toul, alors qu'il rentre de permission en décembre 1916. Dans toutes les gares du parcours, «des poilus énervés s'amusent au détriment du personnel des gares », remarque-t-1l, hurlant et saisissant les casquettes des employés, au point de terroriser les chefs de gare qui « ne paraissent plus ». «Le chant du chef de gare retentit à chaque départ », ajoute-t-il. En gare de Toul, la scène prend une allure qu'il juge « pittoresque » :

9- Louis Guilloux (1899-1980), Le Sang noir, Paris, Gallimard, 1935, 435 p.

10- Michel Bozon, Les Conscrits, Paris, Berger-Levrault, 1981, 156 p.

11- Service historique de l'Armée de terre (SHAT), 16N2401, Note n`15093 du GQG $1^{\text {er }}$ Bureau pour les Armées, 24 décembre 1915. 
«Au départ [...], le chef de gare poursuit un poilu qui lui a enlevé sa casquette. Le train s'ébranle sous des acclamations, tandis que la casquette sombre et dorée est agitée triomphalement à la portière $^{12}$.»

En 1916, l'habitude de tourner en dérision le personnel ferroviaire est bien installée, et se manifeste en particulier à travers la chanson Il est cocu, le chef de gare. Celle-ci est fréquemment évoquée par les sources combattantes, pour lesquelles elle est si connue qu'il n'est pas besoin de l'évoquer autrement que par son titre ou par l'expression « la chanson du chef de gare $»^{13}$. On ne connait que les paroles de la version originale, qui donnent cependant une idée de son esprit moqueur et de l'incitation implicite à la rébellion qu'elle contient :

«J'étais l'autre jour dans l'train d'plaisir

Avec ma femme et mes trois gosses

Ma belle-mère, l'ami Casimir

On voulait s'en payer une tranche

Le wagon était plus que plein

Au départ jugez d'notre colère

Au lieu d'être dix on était vingt

Alors j'appelle par la portière: Chef de gare! Chef de gare!

Aussitôt v'là qu'à l'unisson de tous les côtés on répond :

Refrain:

Il est content le chef de gare

Il est content le chef de gare

Il est près d'sa femme qui vient d'accoucher (bis)

Ohé ! Ohé ! Qui vient d'accoucher.

Casimir qu'est pas endurant

Discute avec une grosse commère

Alors dans le compartiment

On s'colle des gnons sur la caf'tière

Et le train s'arrête brusquement

À peine au milieu du voyage

Pendant plus d'une heure on attend

En criant, braillant sa détresse : Chef de gare! Chef de gare!

Pour passer l'temps qu'on trouvait long,

On gueulait dans tous les wagons :

Refrain

12- Disponible sur le site: www.latecoere.com/carnet/, Carnet de route de Louis Désalbres (1899-1959), 28 et 30 décembre 1916.

13- «La Chanson du chef de gare», L’Echo des Gourbis, n 22 (novembre 1916), p. 2. 
(En face ?) à la première station

On va réclamer l'prix des places :

L'Etat n'rembourse jamais d'pognon

Dit un employé plein d'audace

Là-d'ssus on tombe sur l'matériel,

On bouleverse tout dans la gare

Puis l'on chahute l'personnel

Tout en gueulant dans la bagarre : Chef de gare! Chef de gare!

Les agents nous mènent au violon

Et tout l'long du ch'min nous chantions :

Refrain $^{14} . »$

Son usage est si courant en 1916 que les autorités militaires décident alors de l'interdire. Son ton bon enfant prend en effet dans le contexte militaire une dimension subversive évidente : le chef de gare n'est-il pas le bras technique du commissaire militaire ? La "presse du front» se moque de cette censure, à l'image du Filon, qui dénonce l'arbitraire et l'inefficacité du veto :

«À la vérité, ce refrain n’était pas bien méchant, [...] mais c'est chose connue qu'il exaspère les chefs de gare. [...] La guerre parait-il, a aggravé les choses. Une tradition s'était créée. A peine un train s'arrêtait-il quelque part qu'aussitôt le chef de gare avait, ne le voulût-il pas, son esprit ramené en musique vers un sujet généralement considéré comme délicat et d'ordre privé. [...] Défense expresse a été faite aux permissionnaires de leur appliquer, même en chantant, cet adjectif qu'on trouve dans Molière, qu'on entend à la Comédie-Française, mais qu'on ne prononce pas en bonne société [...].

Les soldats ne chanteront plus qu'il est.... comme vous dites! Ils chanteront qu'il ne l'est pas. Mais peut-être cela suffira-t-il pour qu'il soit satisfait - ce qui montrerait qu'à tout prendre, il a bon caractère ${ }^{15}$.»

La chanson, dont les paroles sont détournées, continue en effet sa carrière après 1916 : « Mais si l'chef de gare est cocu, Faut pas l'gueuler par les portières », déclare une nouvelle version publiée par Rigolboche en juin 1916, tandis que Il est cocu, publiée par Le Diable au Cor en janvier 1917, montre les permissionnaires narguant silencieusement le chef de gare, substitut symbolique du commissaire militaire, qu'il est peut-être trop risqué de ridiculiser ainsi ${ }^{16}$. À partir de l'année 1916, la dimension

14- «Il est content le chef de gare », op. cit.

15- « Le chef de gare et les permissionnaires », Le Filon, $\mathrm{n}^{\circ} 2$ (20 mars 1917), p. 2.

16- «Conseils aux permissionnaires», Rigolboche, $\mathrm{n}^{\circ} 51$ (30 juin 1916), p. 6 ; «Il est cocu ", Le Diable au Cor, $\mathrm{n}^{\circ} 33$ (15 janvier 1917). 
subversive des relations des permissionnaires et du personnel ferroviaire s'affirme à travers une forte critique des rapports hiérarchiques que l'on tente de leur imposer par la discipline militaire et qui ne sont pas justifiés par une compétence technique spécifique.

Les permissionnaires véhiculent un discours sur le rôle du personnel des chemins de fer pendant la guerre qui soulève le problème de son incompétence. Alors que le départ en permission est a priori un facteur de joie pour les combattants, quand le personnel des gares est évoqué, c'est toujours dans le cadre d'une critique du système ferroviaire. On est loin alors de la mythologie ferroviaire du progrès, de la vitesse et de l'espoir présente dans les représentations au début du siè$\mathrm{cle}^{17}$. De façon schématique, on peut dire que dans l'esprit des combattants, les permissions connaissent deux plaies : le voyage en train, et le fait de devoir retourner au front.

Les employés de planton sont stigmatisés pour leur incompétence et leur manque de respect à l'égard des permissionnaires. On l'observe dans Les Boyaux du 95', qui décrivent ainsi l'accueil qui leur est réservé dans les gares parisiennes : « nous voici devant l'entrée des quais tendant à l'employé affairé notre pauvre chiffon de papier, qu'il s'empresse d'estampiller, de noircir d'un geste sec, rageur presque, au petit bonheur ${ }^{18}$. " L'Écho des Marmites conseille avec cynisme aux permissionnaires en octobre 1916 : «ne mets jamais en doute l'indication d'un employé de gare qui par essence est infaillible »; " soumets-toi. ${ }^{19}$ » Dans son roman Une Permission de détente, publié en 1919, Paul VaillantCouturier associe de manière emblématique la sécheresse des employés de la gare de l'Est au sentiment d'abandon du permissionnaire solitaire qui débarque du front ${ }^{20}$. Rarement évoqués, les conducteurs font cependant les frais en juin 1917 d'un trait d'humour du Voltigeur, qui décrit les manœuvres incessantes et absurdes des trains : " Il est bath, l'mécano, y veut pas que l'copain dégueule tout l'temps à la même place », lance un permissionnaire à ses camarades ${ }^{21}$.

17- Marc Baroli, Le Train dans la littérature française, éditions NM, 1969, 493 p. Voir aussi Wolfgang Schivelbusch, Histoire des voyages en train, Paris, Le Promeneur, 1990, $252 \mathrm{p}$.

18- «Retour de permission », Les Boyaux du 95 , $^{\circ}$ 7, s.d., p. 4-5.

19- L'Echo des marmites, $\mathrm{n}^{\circ} 14$ (20 octobre 1916).

20- Paul Vaillant-Couturier, Une Permission de détente, Paris, Flammarion, 1919, 182 p.

21- «Contes de la Creute: Retour de perm’», Le Voltigeur, $\mathrm{n}^{\circ} 3$ (13 juin 1917), p. 3. 
Cependant, les employés les plus critiqués sont aussi ceux qui ont le plus de pouvoir : au quotidien, les têtes de Turc des permissionnaires sont surtout le chef de gare et son supérieur militaire, le commissaire militaire. L'obligation de se soumettre à leur autorité est très tôt contestée par les permissionnaires car elle n'est pas légitimée par une compétence ou un service approprié du transport ferroviaire. Les commissaires militaires sont souvent mis en cause par les combattants qui leur reprochent des contrôles et des tracasseries incessants, alors même qu'ils sont incapables d'assurer leur mission de transmission des informations sur les horaires et les correspondances et qu'ils sont soupçonnés d'avoir intrigué pour obtenir leur poste. En octobre 1915, Le Diable an cor résume un sentiment partagé : « Un commissaire militaire vous regarde d'un œil soupçonneux et annote votre titre de permission. Mais, pour cela, il faut faire la queue pendant une heure ${ }^{22}$ ! Les officiers ne sont pas plus tendres avec eux, à l'image du commandant Courtès, qui rapporte dans ses Sowvenirs de guerre: "Les commissaires de gare qui devraient savoir quelque chose ne savent presque jamais rien et on est, bien souvent, réduit à attendre des journées et des nuits entières qu'on veuille bien nous indiquer le train qui doit nous reconduire à destination ${ }^{23}$.» Alors que les permissionnaires sont fréquemment en proie au cafard au moment de retourner au front, ils apprécient peu d'errer à la recherche de leur unité, une situation fréquente pendant toute la guerre et aggravée pendant les périodes de mouvement, notamment en 1918. Si les sources combattantes insistent sur le statut militaire du personnel des gares, c'est souvent pour souligner le caractère déplacé de leur pouvoir:

« Enfin, voici la Gare Régulatrice. Enfin! Vous croyez pouvoir descendre? Vous vous imaginez pouvoir prendre un café, dans un quelconque buffet. Quelle erreur, Monsieur ! Un employé de la Compagnie de l'Est, flanqué d'un adjudant d'un régiment indéfinissable, se promène sur la voie : "Que personne ne descende ! On va glisser le train sur une voie de garage !..." Deux heures après vous êtes encore au même endroit... Où sont les W.C. ? Ca y est, vous avez le droit, ou plutôt le devoir de descendre... Par corps d'armée et par directions, l'adjudant, flanqué d'un sergent, vous place en ligne. Puis le sergent, flanqué d'un caporal-secrétaire, vous indique l'heure de départ du train qui vous conduira à Paris... ${ }^{24} »$

22- «Les tribulations d'un permissionnaire », Le Diable au cor, $\mathrm{n}^{\circ} 13$ (octobre 1915), p. 3. 23- SHAT, 1KT20, Souvenirs de guerre du commandant Courtès, avril 1918.

24- «Les tribulations d'un permissionnaire», art. cit. 
Le journal Le Diable au Cor publie plusieurs articles dénonçant le rôle du personnel ferroviaire dans des trajets longs et pénibles, placés sous le signe de l'absurde, dans des récits où le permissionnaire prend la figure d'un ingénu, ballotté de gare en gare au gré des ordres contradictoires des commissaires militaires. Dans une «lettre ouverte au Directeur de la Compagnie des chemins de fer de la Somme au patelin », le journal dresse en octobre 1916 un portrait charge de la situation ferroviaire, incriminant sans les nommer le chef du $4^{e}$ Bureau de l'état-major en charge des transports auprès du ministre de la Guerre, et Claveille, alors sous-secrétaire d'État aux Transports ${ }^{25}$. Pour autant, la mise en cause des responsables de la politique ferroviaire est rare, notamment parce qu'elle est interdite par la censure et doit emprunter des voies détournées pour se manifester, comme ici. L'interdiction de la chanson du chef de gare en 1916 a peut-être incité les critiques à trouver un autre exutoire que la carnavalisation de l'ordre établi.

La crise du moral du printemps 1917 trouve en effet dans les gares et les trains un terrain d'expression privilégié, se traduisant par des graffitis et des dégradations dans les voitures, du chahut, des insultes et des coups envers le personnel ferroviaire. Fin mai, le permissionnaire Louis Désalbres décrit une gare parisienne « très houleuse » où le train du retour s'ébranle dans « un vacarme de cris et de chants séditieux $»^{26}$. À la même période, l'inspecteur de la Commission du réseau Nord dénonce «l'impuissance du commissaire militaire, du chef de gare et des agents de tout ordre à faire obéir les permissionnaires ", alors que ceux-ci « ouvrent les portières des trains en marche, s'assoient sur les marchepieds, montent sur les toits des voitures, courent à moitié nus le long des voies $»^{27}$. Le 8 juin 1917, en gare de Chantilly, des permissionnaires jettent des pierres sur les locaux et le chef de gare, après une erreur d'acheminement d'un train ${ }^{28}$. Quelques jours plus tard, un officier supérieur en retraite témoigne de ce qu'il a observé au cours d'un voyage dans l'Ouest :

25- « Propos d'un de la "Verse". Lettre ouverte à M. le Directeur de la Compagnie des Chemins de fer de la Somme au patelin », Le Diable au cor, $\mathrm{n}^{\circ} 31$ (5 octobre 1916), p. 3. Voir aussi «Les tribulations d'un permissionnaire », art. cit.

26- Désalbres, op. cit., 22 mai 1917.

27- SHAT, 16N2851, Commission du réseau Nord, note pour la direction des chemins de fer, 15 mai 1917.

28- SHAT, 16N2405, Rapport de l'inspecteur principal de la $1^{\text {te }}$ région au commissaire technique de réseau à Paris-Nord, 8 juin 1917. 
« [...] J'ai vu un contrôleur de la Compagnie des chemins de fer jeté à bas d'un wagon de $1^{\text {re }}$ pour avoir voulu faire exécuter les ordres du ministre de la Guerre relatifs aux classes à occuper par les militaires en congé. J'ai entendu narguer blaguer et insulter de malheureux gendarmes de planton dans la gare pendant que le train se mettait en marche $[\ldots]^{29}$.»

On pourrait citer d'autres incidents, qui montrent que le personnel des gares est fréquemment pris à partie par les permissionnaires qui se déchainent dès qu'un train est en retard ou stationne longtemps en gare, et craint les soldats, si bien que, dans certains gares, des rapports soulignent qu'il se cache lors du stationnement des trains, parfois imité par les «représentants de l'autorité $»^{30}$. Il faut en effet souligner que le tumulte des permissionnaires, même le plus violent, a souvent des causes objectives qui reflètent les dysfonctionnements du transport des permissionnaires depuis 1915, qui s'est aggravé depuis la fin mai 1917 avec l'augmentation subite et anarchique du taux de permission.

En effet, totalement improvisé, le transport des permissionnaires ne connait pas le caractère prioritaire des transports stratégiques. Le voyage s'effectue dans des trains militaires au statut de trains de marchandises, soumis à des règles spécifiques de composition, de vitesse, de charge et de manœuvre. Les trains spéciaux de permissionnaires instaurés à partir de septembre 1915 subissent ces contraintes, qui se cumulent avec l'usure précoce du matériel pour rendre les voyages lents. On comprend que les permissionnaires aient pu prendre pour une provocation la dénomination d'« express » ou de «rapide » que le haut commandement leur attribuait. Par ailleurs, le manque de personnel se traduit par la dégradation et la saleté des voitures, qui sont aussi mal éclairées et peu chauffées, même en hiver. À l'inverse, en été, la lenteur des voyages les rend éprouvants, ce qui a sans doute joué un rôle lors du printemps caniculaire de 1917, pendant lequel les permissionnaires se sont signalés par leurs beuveries et le débraillé de leurs tenues. Les problèmes de régulation sont constants depuis 1915, liés aux fluctuations du taux de permission et au manque de coordination entre les

29- SHAT, 16N2405, Lettre de l'officier supérieur Bourly au général en chef, 13 juin 1917.

30- SHAT, 16N1543, Rapport du chef d'état-major de la III Armée au général en chef, 7 août 1917. Pour une analyse générale de la crise du printemps 1917 : Guy Pedroncini, Les Mutineries de 1917, Paris, PUF, 1996 (1967), 328 p. ; Leonard V. Smith, Between mutiny and obedience: the case of the French Fifth Infantry Division during World War I, Princeton, Princeton University Press, 1994. Voir aussi le mémoire d'André Loez, «Les mutins de 1917. Répertoire d'une action collective », IEP de Paris, 1997, 218 p. 
militaires et les instances techniques. En conséquence, les trains sont bondés et beaucoup de permissionnaires voyagent debout. C'est particulièrement le cas à partir de la fin de l'année 1916, où la mise en place de la Charte des permissionnaires garantit à chacun le droit à une permission tous les quatre mois, sous réserve d'opérations militaires. Alors que, depuis l'été 1915, le nombre de permissionnaires s'adaptait à la capacité ferroviaire, on assiste alors à une diminution de la flexibilité des permissions. En moyenne, le trafic quotidien des permissionnaires sur le réseau s'élève jusqu'à la fin de la guerre à 45000 . Enfin, les trains circulent sans que la sécurité des voyageurs soit toujours assurée, comme l'ont révélé plusieurs accidents graves, tel le déraillement le 13 décembre 1917 d'un train de permissionnaires à Modane qui causa la mort d'environ 800 permissionnaires dans ce qui reste à ce jour le plus grave accident ferroviaire français. Une seule locomotive tirait deux trains et le système de freinage était inadapté ${ }^{31}$.

Les conditions de transport des permissionnaires sont donc au total très pénibles, sans cesse dénoncées par les combattants et connues des parlementaires qui s'emploient à les améliorer. Un rapport de la commission de l'Armée de juin 1917, à une époque où les troubles occasionnés dans les trains et les gares incitent à la recherche des facteurs de la crise, est accablant. S’adressant à ses collègues, le député radical Lauraine déclare :

«Vous savez tous qu'il y a à l'arrière des troubles dus aux permissionnaires. Croyez-vous que nous n'ayons pas une part de responsabilité et que nous n'ayons pas à nous reprocher notre incurie? Avez-vous remarqué que la révolte s'étend de l'arrière au front? Une des causes déterminantes, c'est qu'ils sont renvoyés aux armées dans des conditions déplorables; dans un couloir de voiture, on empile jusqu'à 76 hommes ; du bétail serait mieux traité, car on craindrait l'intervention de la LPA ! Et dire que le voyage dure de 12 à 14 et même 16 heures. Si on nous imposait pareil supplice, nous serions pires que les poilus : quant à eux, ils profitent de tous les arrêts pour faire remplir leurs bidons, ils boivent, se saoulent et ne savent même plus ce qu'ils font... ${ }^{32}{ }^{\prime}$

31- Au sujet de cet accident on peut consulter le site www.travel.to/danger. Voir aussi "Grave accident de train à Massy-Palaiseau », Le Petit Parisien, 18 janvier 1917.

32- LPA : Ligue protectrice des animaux. AN, C 7498, PV des séances de la Commission de l'armée, 28 juin 1917. 
À l'époque, le tableau est juste, et s'applique aussi aux trajets du front vers l'arrière. L'image d'une machine sans logique domine d'ailleurs les représentations du voyage des permissionnaires. De manière emblématique, dans Les Suppliciés, un roman de René Naegalen paru en 1927 qui mêle souvenirs et fiction, le voyage épuise le héros : «que le temps lui dure, dans ce train sans horaire, aux arrêts inexplicables, et qui se traine lentement ${ }^{33}$. » Quel que soit le type de document, les permissionnaires semblent confrontés à un système de transport inefficace et aliénant, dont les cheminots font les frais bien qu'ils ne soient pas jugés responsables des problèmes. On en trouve un exemple significatif dans les carnets de route du sergent Granger, qui raconte son voyage de l'Eure à l'Argonne en mars 1917 :

"Je voyage toute la journée en chemin de fer pour arriver à Jonchery après avoir passé par Epernay. Je passe la nuit dans un marabout, et je vais à Montigny, de là, on me renvoie à Damery où je passe la journée, j’ai fait le voyage en auto heureusement car par le train c'est la brouette : retrouverai-je mon régiment ${ }^{34}$ ? »

L'anonymat du « on » est à l'image de la machine folle, absurde et inadaptée qu'est le transport ferroviaire des permissionnaires. La pénibilité du voyage en train, associée au tumulte traditionnel des permissionnaires, a donc joué un rôle dans l'exacerbation des passions et le ressentiment des combattants, qui voient les civils voyager dans des trains commerciaux, alors qu'eux-mêmes ont l'obligation d'emprunter les trains spéciaux et d'emprunter des classes définies en fonction de leur grade et de leur mérite militaire. On connait mal les sentiments qu'une telle situation a pu engendrer parmi les cheminots, pris entre la nécessité d'assurer le service dans une improvisation permanente, de faire respecter la discipline militaire pour certains d'entre eux, et subissant au quotidien les tracasseries des permissionnaires qui les considèrent comme des « embusqués ». Leur attitude semble avoir varié entre deux pôles : l'abus de pouvoir et la passivité. L'écrivain-ouvrier Pierre Hamp explique ainsi l'autoritarisme des commissaires militaires, boucs émissaires de tout un système : « quand des hommes subissent la honte d'être inutiles, la tracasserie devient leur métier. » Pour lui, cette situation est l'effet du bouleversement des hiérarchies ferroviaires pendant la guerre : «la guerre met au-dessus du chef de gare le commissaire régulateur et

33- René Naegelen, Les Suppliciés, Paris, La Baudinière, 1927, 316 p., p. 74.

34- Roger Girard (éd.), Carnets de guerre du sergent Granger 1915-1917. La Grande Guerre vécue et racontée au jour le jour par un paysan de France, Montpellier, Université Paul-Valéry, ESID, 1997, 218 p. 
le commissaire militaire, qui doivent se donner l'apparence de compter pour quelque $\operatorname{chose}^{35}$. » Le personnel des gares semble de son côté avoir montré peu de zèle dans la surveillance des permissionnaires. Dans la plupart des gares de la région parisienne, où transitent la majorité des trains de permissionnaires, le nombre de combattants en transit chaque jour est tel que les employés et le service d'ordre militaire sont bien en peine de contrôler tous les titres de permission et de surveiller les issues des gares $^{36}$. Certains se sont contentés de découper les coupons de transport, sans vérifier la régularité des titres, comme on l'observe lorsque les permissionnaires finissent par arriver, en toute illégalité, dans les gares terminus parisiennes ${ }^{37}$. Pour gagner plus rapidement la capitale, les permissionnaires empruntent les trains commerciaux qu'ils croisent ou finissent le voyage à pied, s'échangent les ruses pour sortir en fraude des gares de banlieue ou pour faire timbrer leur permission plus tard que prévu, gagnant ainsi quelques jours de congé. Les retardataires rejettent la responsabilité sur le personnel des gares, mais il est difficile de parler de complicité active, tant la tâche dépasse les effectifs. C'est ce que rappelle le commissaire technique de Noisy-le-Sec en janvier 1918 lorsqu'il est confronté aux critiques des autorités militaires ${ }^{38}$. Le sentiment de culpabilité d'une corporation qui échappe largement au service en armes a aussi pu jouer un rôle dans le manque de zèle des employés à contrôler des combattants placés au premier rang de l'économie du sacrifice. Il faudrait utiliser les archives des compagnies pour savoir si des enquêtes internes ont eu lieu, et quels ont été leurs résultats.

Les représentations de la rencontre des permissionnaires et des cheminots sont donc placées sous le signe de l'inversion des hiérarchies statutaires, qui tend à dégrader l'image de la corporation, alors même qu'en termes de transports de concentration ou de ravitaillement les chemins de fer semblent donner satisfaction. Affectés spéciaux, les cheminots entrent dans la catégorie des " embusqués ", mais leur utilité n'est pas remise en cause lorsque le système des transports de permissionnaires donne à peu près satisfaction. Lorsque celui-ci entre en crise à la fin de l'année 1916, la tentation de critiquer l'ordre existant

35- Pierre Hamp, La Victoire mécanicienne, Paris, Gallimard, coll. NRF, 1920, 167 p., p. 131.

36- SHAT, 16N2851, Rapport sur les effectifs du service d'ordre des gares de permissionnaires, GAN, 20 juillet 1917.

37- Par exemple lorsqu'ils devaient se rendre en province ou lorsqu'ils sont en retard.

38- SHAT, 16N2851, Lettre du commissaire technique de Noisy-le-Sec au commissaire régulateur, 10 janvier 1918. 
prend le dessus, et c'est ce qui s'exprime dans les gares parce que l'interdit frappe moins les cheminots au statut hybride, mobilisés à l'arrière, que la hiérarchie militaire qui est présente au front, mais aussi dans les gares et les trains. L'image d'une communauté ferroviaire embusquée et incompétente permet aussi aux combattants de s'auto-définir, a contrario, comme une communauté virile dont la domination s'exprime symboliquement par les pratiques de transgression de l'ordre militaire dans l'espace ferroviaire. Le sentiment que la permission commence au départ du front, et non à la gare d'arrivée, a aussi pu jouer, s'opposant à la conception des autorités militaires qui cherchent à faire régner la discipline tout au long du voyage dans un contexte de sous-encadrement chronique qui rendait sans doute ce pari impossible. Enfin, les critiques portant sur les défaillances logistiques font naitre ce qui s'apparente à un discours politique sur le service public dû par l'État aux combattantscitoyens. 